\title{
Physiological Aspects of the User's Immersion in the Virtual Reality Environment on the Example of Creating a Hardware-Software Complex for Smell Transmission *
}

\author{
Artem Smolin ${ }^{[0000-0001-7242-6471]}$, Konstantin Malyshev ${ }^{[0000-0002-8455-5876]}$, Anna Spiri- \\ donova $^{[0000-0003-0724-0405]}$, Andrey Mironov ${ }^{[0000-0001-5336-8800]}$, Aleksei Shchekoldin ${ }^{[0000-}$ \\ 0003-0824-8011], Pavel Vorobyev ${ }^{[0000-0002-7447-0502]}$ \\ ITMO University, Saint-Petersburg, Russia \\ smolin@itmo.ru, malyshevkoditmo.ru, spirannalist.ru, \\ mr_blum@mail.ru, ashchekoldin@itmo.ru, passeridaepc@gmail.com
}

\begin{abstract}
This article discusses the use of prototypes of various technological devices to use odor sensory channel for higher immersion in virtual reality environments. Based on the results of research conducted by the teachers and students center of Usability and Mixed Reality at ITMO University, a prototype of a device for transmitting odors was developed. The article presents the concept and technological scheme of this device. The developed hardware and software complex consist of special platform based on a microcontroller (Arduino Nano) and a control device. The control device was built on the architecture of ARM, with the possibility of wireless connection. To control the air flow and odor supply from the tanks, it was selected a water pump, connections to the tanks is provided by with the help of special valves with a crane. In addition, for this software and hardware complex, a special three-dimensional location was modeled, as well as the design of the backpack and the scheme for its attachment.
\end{abstract}

Keywords: Virtual Reality; Hardware and Software Complex; Three-Dimensional Modeling; Device for Transmitting Smells.

\section{Introduction}

In the work "On the Origin of Species by Means of Natural Selection, or the Preservation of Favoured Races in the Struggle for Life ", Charles Darwin stated, that natural selection acted for the benefit of every being, so the physical and mental qualities tended to develop in the direction of perfection [1].

Applying this statement in the aspect of technologies of virtual and augmented reality, it is possible to presume that developing these technologies, we strive for the most complete ("perfect") existence in cyber reality, both mentally and physically. This

Copyrightc $(2020$ for this paper by its authors. Use permitted under Creative Commons License Attribution 4.0 International (CC BY 4.0).

* Supported by the Government of Russian Federation (Grant 08-08) 


\section{A. Smolin et al.}

means that when we put on a virtual reality headset, we isolate ourselves from the real world and completely immerse ourselves in the computer reality. At this stage of technology development, this is possible through by means of the use of sensory channels of perception of a human being.

The first and main channels of transition from reality to the virtual world were visual and audio channels. This already allows the partial destruction of the "fourth wall" through the relative realism of virtual locations. An example of this in virtual reality is the dualism of the user, on the one hand he can stay himself projecting his mental reflection into the computer world, or on the other hand he can try on the role of a character with specific qualities that may contradict the user, but he is ready to accept the rules of the game [2].

The use of visual and audio channels is incomplete, since the user while being in virtual reality does not feel the tactility of the virtual world (so far only the vibration of controllers and prototypes of suits and gloves for virtual reality), he is distracted by extraneous odors that do not correspond to the virtual space where he is located.

The first devices that activated various sensory channels of user perception for viewing various content were created in the middle of the twentieth century. A pioneer in this field can be considered American cinematographer and inventor Morton Heilig, who described the concept of multi-sensory theater in the article "The Cinema of the Future".

In 1962, Morton Heilig built a prototype of his immersive, multi-sensory, mechanical multimodal theater and named it "Sensorama" [3]. Sensorama consisted of multi sensors that could make a chromatic film that previously recorded to be augmented by clear sound, smell, the wind and related vibration [4].

In those days it looked impressive, but Morton Heilig did not have what we possess now - possibilities for a complete immersion in the computer location.

Therefore, having based on the experience of creating such devices, we have already tried to create prototypes of software and hardware systems with the goal to reach the complete mental and physical immersion of users in virtual reality environments.

Having analyzed the philosophical, technological and social aspects of this problem, the staff of the Center of Usability and Mixed Reality at ITMO University, together with the students of the Faculty of Software Engineering and Computer Systems, set themselves the task of creating a prototype device for transmitting odors while the user is in environments of virtual reality.

This prototype is planned to be used in conjunction with any virtual reality headset. The prototype must possess qualities that are convenient for inexperienced users, such as: minimum weight, ease of use and low production cost.

There was made a fundamental decision not to place the device directly on the virtual reality headset, but to make it wearable in order to reduce the load on the headset (which is located on the user's head) and provide the necessary safety measures. 


\section{Researches, technologies and prototypes of olfactory virtual reality systems}

Olfactory virtual reality systems can be used in all spheres of activity: military, industrial, educational, entertainment, etc.

In 1960 the Smell-O-Vision system was created, which injected 30 different odors into the movie theater seats at different points during the film [5].

The article "Olfactory Display Prototype for Presenting and Sensing Authentic and Synthetic Odors" demonstrates a study on how productive an olfactory display prototype can be used to affect participants' cognitive and emotion related responses to audio-visual stimuli [6].

For the experiment, an olfactory display prototype was created, which included an air compressor (HBM AS-48) and plastic Teflon coated tubes to transfer scented air to a mask worn by participants. Specially selected thematic materials were shown to the participants in the virtual reality headset Oculus Rift DK2.

The main goal of the experiment was to investigate if participants' cognitive and emotion-related responses differ between authentic and synthetic scents.

Twenty-nine volunteers took part in the experiment. Lemon peel was used as an authentic scent, limonene was used as a synthetic scent.

Three videos were used as visual material. Two of them matched the smells - lemon spray and lemon picking, and one was incongruent - potato peeling.

After the demonstration, the participants were asked to rate the intensity of the odor and pleasantness and arousal of the viewing experience on three nine-point bipolar scales that varied from -4 (mild, unpleasant or calm) to +4 (strong, pleasant or aroused).

As a result of the experiment only lemon peel was smelled in $100 \%$ of the cases. Limonene was detected almost equally well suggesting that almost all the participants were able to smell the odor. Odors were rated as more intense when the video was congruent with the odor [6].

The results of this study have shown that odors combined with thematic video materials have a significant impact on enhancing perception and, as a result, on a more authentic immersion in the virtual reality environment.

The article «Season Traveller: Multisensory Narration for Enhancing the Virtual Reality Experience» shows multisensory hardware and software complex, including olfactory and haptic components (Figure 1) [7].

Season Traveller - a wearable VR system based on virtual reality headset Samsung Gear VR that integrates thermal, wind and olfactory stimuli. The device consists of a set of fans (wind), Peltier elements (thermal) and micro air-pumps (olfactory), which are controlled by commands that are sent via Bluetooth.

Before developing Season Traveller, the creators started from two basic principles:

1. Minimal violation of user safety and comfort

2. The design should not prevent users from accessing the virtual reality headset

Toolkit 


\section{A. Smolin et al.}

In addition, to redistribute the weight of the structure, it was decided to distribute the components between the front and back of the user's head (Figure 1).

This project is interesting because in addition to the device itself, specifically for it there was developed a VR game that takes users on a journey through four different virtual seasons in a mystical hot-air balloon ride [7].

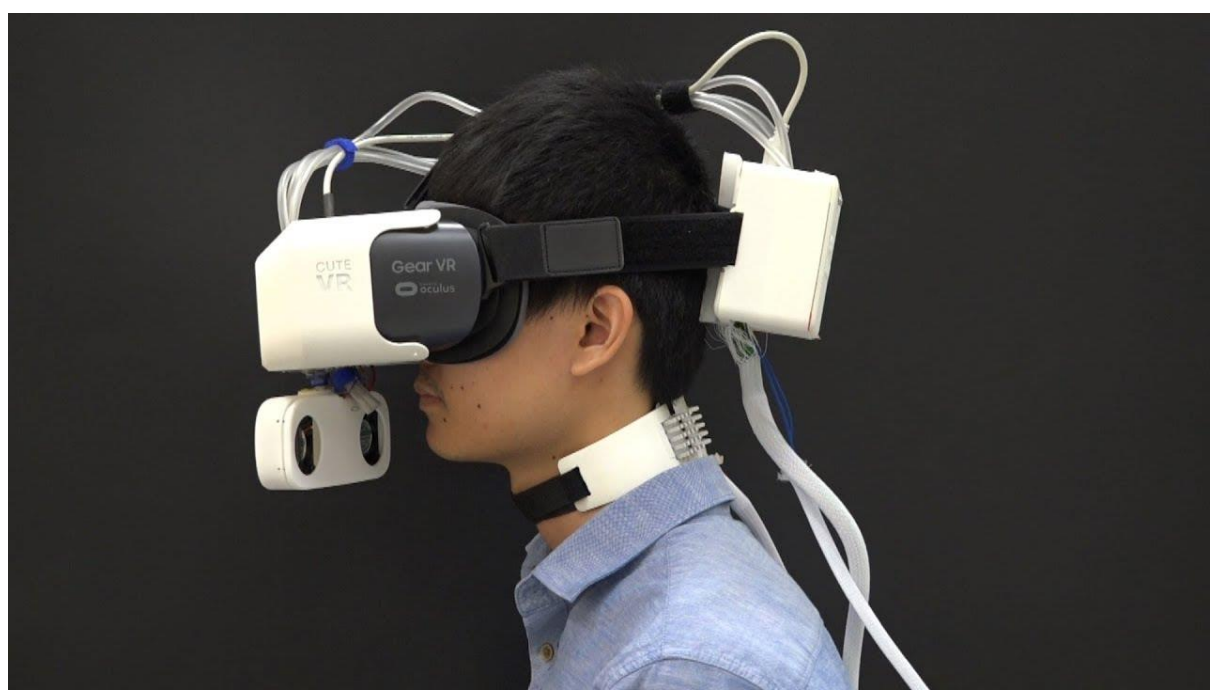

Fig. 1. Season Traveller

Three preliminary studies were conducted to create olfactory stimuli:

3. The types of olfactory stimuli

4. The stimuli delivery method

5. The perceived intensity level of the stimuli

To select the scents for each season, there was created a special experimental smelling chamber that featured three types of smell-emitting mechanisms: air-pump, microfan, and piezodiffuser.

As a result of experiments with twenty-seven respondents the following odors were selected: Spring - Jasmine scent; Summer - Lemon scent; Autumn - Cinnamon scent; Winter - Mintscent.

Olfactory Simulation Module consists of four air-pumps that are each connected to a separate chamber containing essential oil (Jasmine, Lemon, Cinnamon and Mint). Each of the air-pumps is connected to a separate $45 \mathrm{~cm}$ medical-grade polyurethane tube that runs over the top headband of the virtual reality helmet, delivering scented air near the nose of the user [7].

The authors decided to take a similar technological scheme as a basis, except for the system for placing the odor module, not on a virtual reality headset, but as a separately wearable device. 
To study perception, an experiment was conducted with twenty respondents, during which various biometric data were collected and analyzed from medical sensors - Heart Rate and Electrodermal Activity.

In addition to the analysis of medical parameters, users were questioned. This questionnaire consisted of twenty-three questions that were adapted from Witmer and Singer's Presence Questionnaire and six questions from the Game Experience Questionnaire (GEQ) [7].

As a result of the conducted research, it was found that adding any modality improves the sense of presence in relation to the traditional audiovisual experience. Providing a combination of these modalities produces a further significant improvement in the aspect of immersion in virtual environments.

In addition to research and development, commercial prototypes olfactory virtual reality systems are created.

One of the prototypes is the VAQSO VR (Figure 2), a compact device for transmitting odors integrated with virtual reality environments. The device can be attached to all major virtual reality headsets and it gives a user an opportunity to enjoy fifteen different scents from five replaceable cartridges [8].
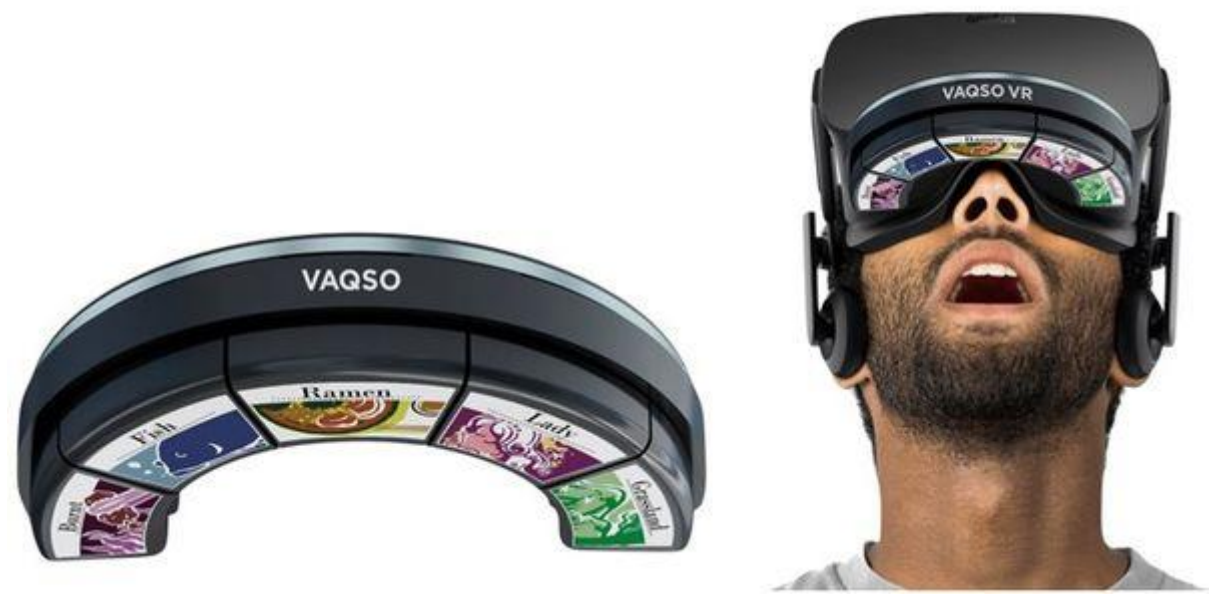

Fig. 2. VAQSO VR Device

One of the most successful devices is the Feelreal Sensory Mask (Figure 3) [9]. The mask attaches to the viewer's face and to one of the virtual reality headsets, and then a scent generator with nine individual aroma capsules releases odors into the nose area (simulates hundreds of smells).

This device comes with the Feelreal SDK, which allows developers to add various individual scents to their projects. In addition, the special FeelReal Player gives an opportunity to use different flavors while watching movies [10]. 


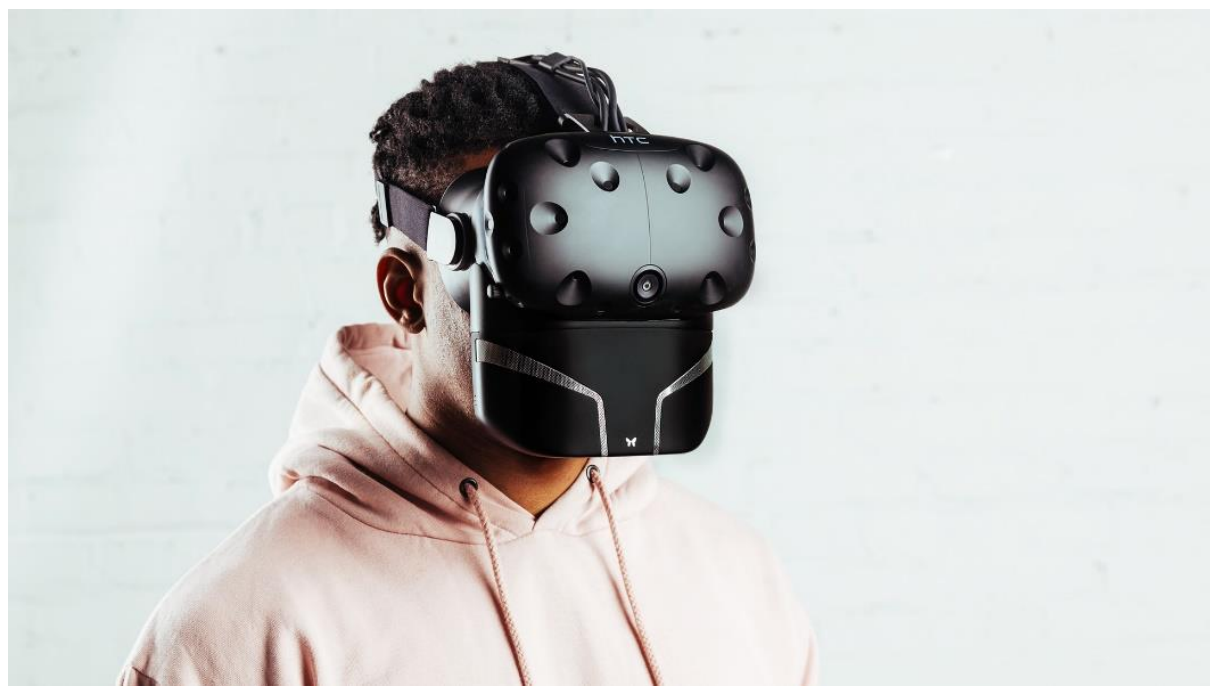

Fig. 3. Feelreal Sensory Mask

\section{Creating a prototype device for transmitting smells in virtual reality environments}

\subsection{Search for a technological solution for the first version of the prototype.}

After analyzing the various studies conducted and prototypes created, a technological solution was chosen based on storing odors in a liquid state and spraying them by means of a system of valves and a system of air pumping through tubes connected to containers with odors.

The main advantages of this approach are:

- Number of available odors

- Control the amount of odor supplied

- Scalability of the system

- Ability to mix flavors with each other

As a result of the analysis of currently available devices, a platform was selected based on a microcontroller and a control device, since this option completely allowed us to solve the tasks with the ability to expand and change parts of the device being created.

To control the air flow and odor supply from the tanks, it was selected a water pump with voltage support up to twelve volts for pumping air through the system. Connections to the tanks were used with the help of special valves with a crane; motors without a gearbox with an operating voltage of five volts were connected to them. 
Physiological Aspects of the User's Immersion in the Virtual Reality Environment... 7

For this device there was chosen microcontroller Arduino Nano because of its simplicity and capabilities, as well as due to compatibility with other, more powerful counterparts. In addition, thanks to this microcontroller, it became possible to expand the necessary functionality without a large expenditure. The control device was built on the architecture of ARM, with the possibility of wireless connection.

As a result of the work done, the following components were identified:

- Control device

- Microcontroller

- Engines

- Pump

Figure. 4 shows the third iteration of the scheme, which indicates the channels of exchange commands, power and the following components of the system:

- The motor driver

- Pump driver

- Power supply

- Voltage converter

- Microcontroller

- Control device

- Pump

- Engine

- Valve

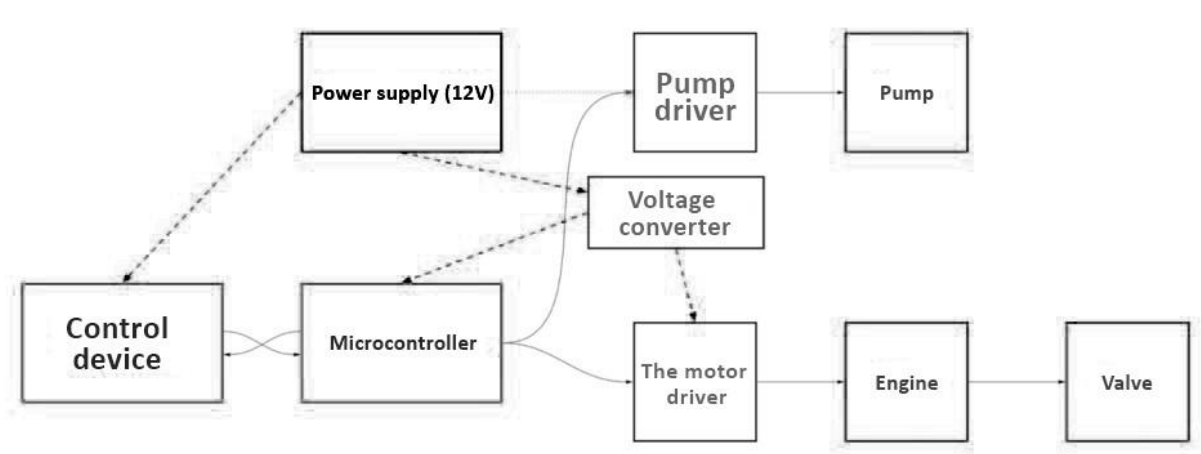

Fig. 4. Device diagram for transmitting smells in virtual reality

The final version of the device diagram, which is presented in Figure 5 shows a complete diagram of interactions between device components and external sources. 


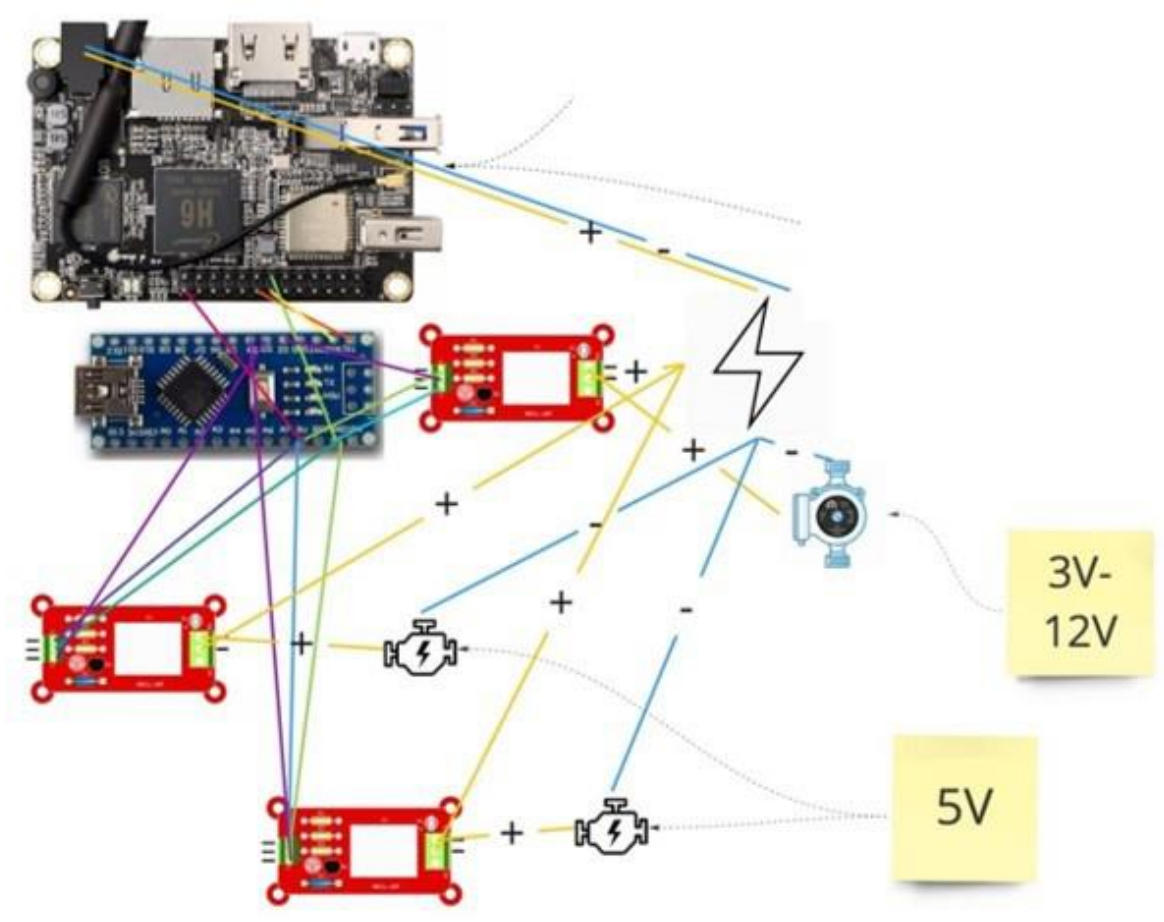

Fig. 5. Component interaction scheme device and external sources

The result of undertaken work was a prototype device with the reproduction of odors in the headset of virtual reality HTC Vive.

\subsection{Research of the first version of the prototype.}

To test the developed prototype, the ready-made virtual location "The Night Cafe - An Immersive VR Tribute to Vincent van Gogh", created by Borrowed Light Studios, was taken. It is a journey into Vincent Van Gogh's The Night Cafe [11]. To enhance the effect of immersion, the smells of coffee and port wine were used, which were typical for these establishments of that time (Figure 6).

The mixing of these two odors took place directly during the activation of the device. Each odor was fed from the source to a separate tube, the mixing of odors took place in an output device located in front of the user (Figure 6).

A group of twenty-four respondents was selected for the experiment, the prototype allowed changing the intensity of odors and initiating odors in the right places by manual control.

Each respondent was allocated thirty minutes to be in the virtual space and fifteen minutes to complete the survey. 
Physiological Aspects of the User's Immersion in the Virtual Reality Environment... 9

List of questions included in the user's survey:

- How real what was happening in the virtual reality headset was for you (from 1 to 10 points)

- Did you feel any discomfort while you were in virtual reality (yes / no)

- Did you feel any restrictions that prevented you from fully immersing yourself in the virtual location (text)

- Did you smell what was happening in front of your eyes (yes / no)

As a result of the survey, most of the respondents noted that they did not feel discomfort and smelled odors together with visual viewing, which allowed them to feel the virtual location more fully.

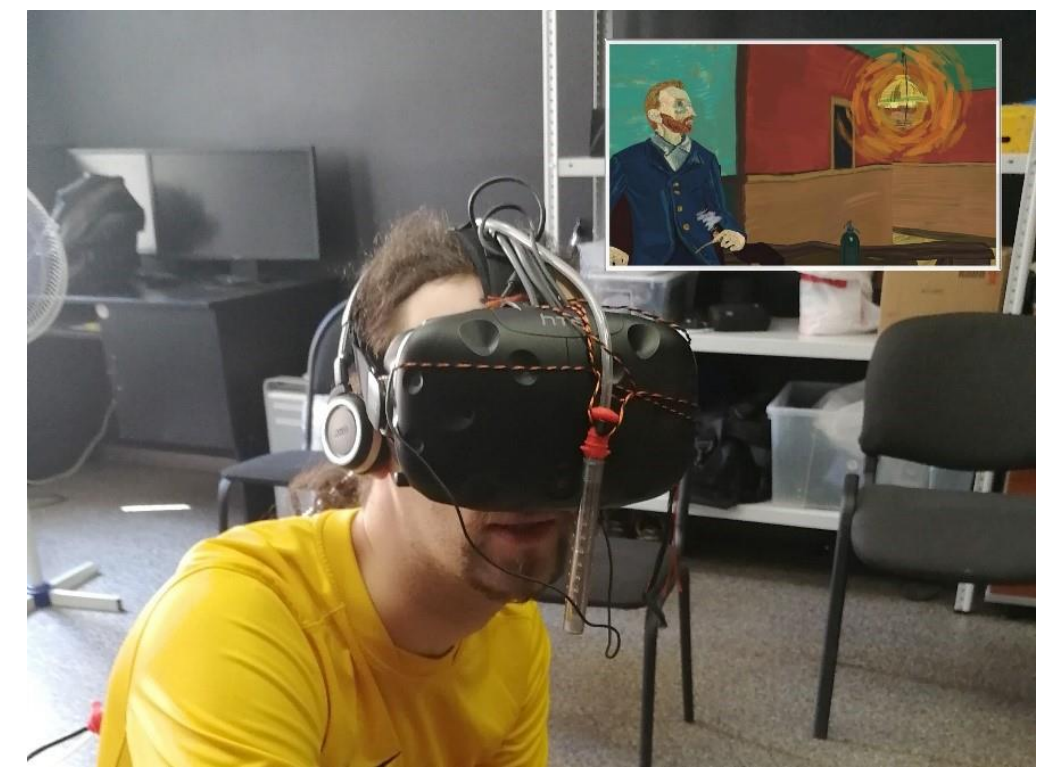

Fig. 6. Prototype of the device with odor reproduction

\subsection{Ergonomics of the device for transmitting smells in virtual reality}

During the process of creating and testing the prototype, parallel work was simultaneously conducted to create a backpack in which this prototype will be placed.

After analyzing the prototypes that are attached directly to the virtual reality headset increasing its weight and shifting the center of gravity of the device, it was decided to make a prototype of the device that the user would carry on his back in a specially designed backpack. Figure 7 shows a sketch of a prototype worn on the back. 
10 A. Smolin et al.
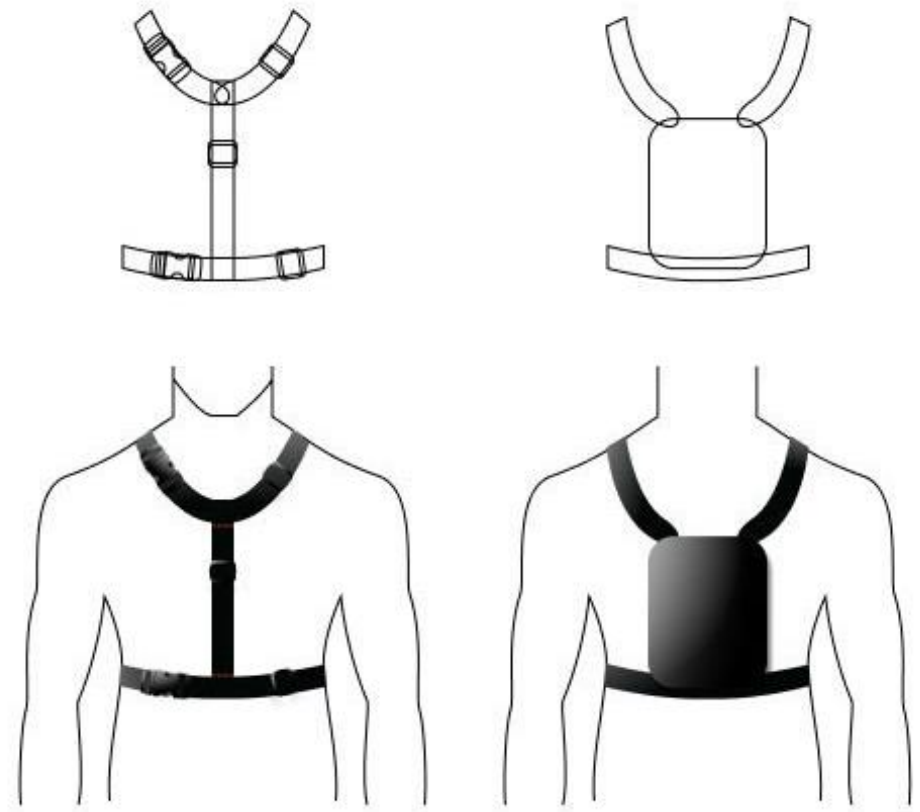

Fig. 7. The scheme of fastening a backpack for prototype device for transmitting smells in virtual reality

A special fixed tube connects the prototype, placed in a backpack, to the device that sprays odors on the headset, which does not affect the weight and center of gravity of any virtual reality headset used. This makes the system more versatile and secure.

\subsection{Virtual location for working with a prototype}

To implement all the capabilities of the device, it was required to develop its own virtual scene, which corresponded the following requirements:

- A special virtual stand for the location of the smell source

- Design of space

- Correct retopology working in various virtual reality headsets

Figure. 8 presents a developed model of the scene with special locations for concentration of smell in high tech style. 


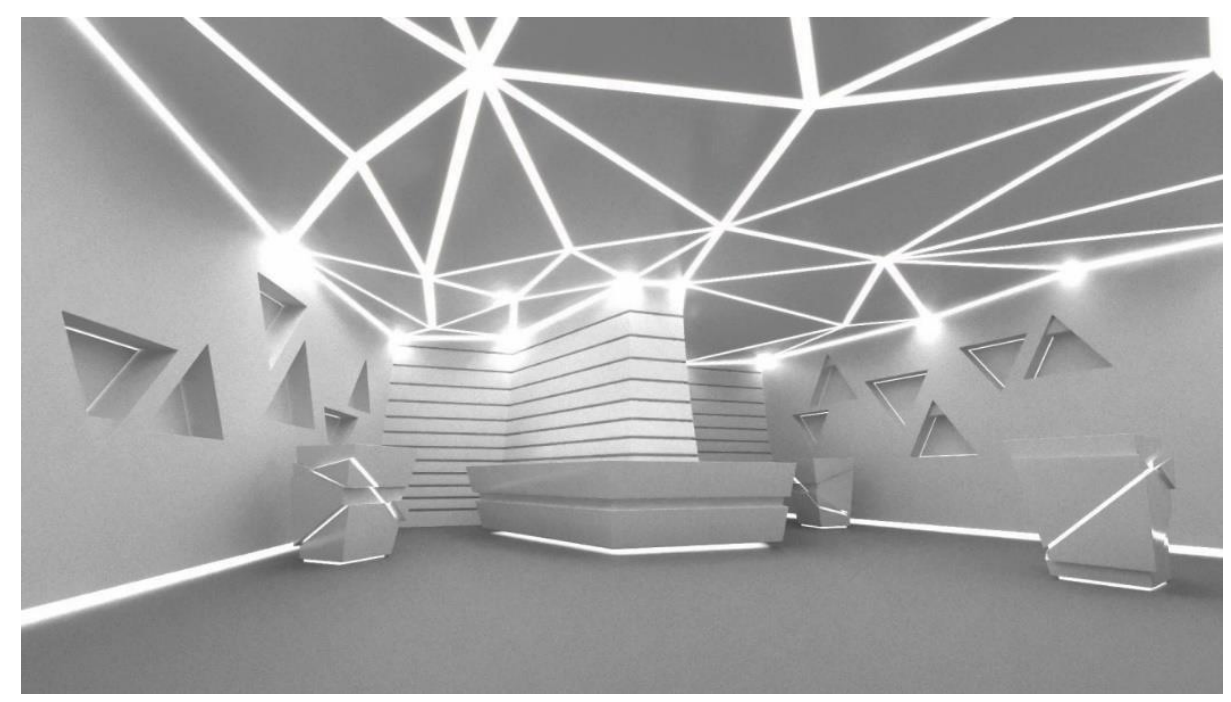

Fig. 8. Virtual location created specifically for the developed prototype device for transmitting smells in virtual reality environments

\section{Conclusions}

The use of virtual reality headsets made it possible to achieve high immersion in computer environments already at the level of visual and audio sensory channels.

It is quite logical that the next steps in the aspect of full-fledged stay in virtual environments will involve the rest of the sensory channels in the process, so at the moment there are a decent number of prototypes of various technological devices, which will later be combined into a single hardware-software complex this would result in making the most authentic and comfortable immersion in virtual reality environments, whether it is a computer game, educational simulation, telepresence system, etc.

As a result of the work done by the staff of the center of Usability and Mixed reality at ITMO University, a prototype of a smell reproduction device for virtual reality environments was developed, which included the following stages:

- Selection of odor reproduction technology based on prototypes analysis

- Technical development of the device

- Selection and creation of various test applications for further research works

The fundamental difference from the considered analogs was the use of a portable device for supplying and mixing odors. According to the authors, this will reduce the load on the headset and provide proper safety equipment (the device cannot fall off the headset, which can harm the user or cause damage to the device).

When creating the second version of the prototype, the tasks were set to solve problems connected with the size of the device and the placement of its components on a single Board. 
12 A. Smolin et al.

Instead of devices OrangePi and Arduino Nano, it was decided to use the Nodemcu device, which will lead to implementation of the functionality of both devices previously used at a more compact size.

Instead of the power supply unit, Will be used a Li-pol battery with the appropriate control controller. Replacing the pressure pump will reduce the number of components and simplified the circuit. As a result of this work, the created prototype will become more compact and functional.

\section{References}

1. Darwin, C.: On the origin of species by means of natural selection, or, The preservation of favoured races in the struggle for life. London: John Murray (1859).

2. Breaking Through the Fourth Wall in Virtual Reality, https://vrscout.com/news/breaking-fourth-wall-virtual-reality/\#, last accessed 2013/12/21.

3. The Sensorama: One of the First Functioning Efforts in Virtual Reality, http://www.historyofinformation.com/detail.php?id=2785, last accessed 2011/06/01.

4. Asmaa, A., Lamia, F., Foaud, L.: Environments and System Types of Virtual Reality Technology in STEM: A Survey. (IJACSA) INTERNATIONAL JOURNAL OF ADVANCED COMPUTER SCIENCE AND APPLICATIONS 2017, vol. 8, pp. 77 89. SAI Organization (2017).

5. Steven M. LaValle: Virtual reality, http://lavalle.pl/vr/, last accessed 2019/12/19.

6. Salminen, K., Rantala, J., Isokoski, P., Lehtonen, M., Müller, P., Karjalainen, M., Väliaho, J., Kontunen, A., Nieminen, V., Leivo, J., Telembeci, A., Lekkala, J., Kallio, P., Surakka, V.: Olfactory Display Prototype for Presenting and Sensing Authentic and Synthetic Odors. In: ICMI '18: Proceedings of the 20th ACM International Conference on Multimodal Interaction, pp. 73-77. ACM DL (2018).

7. Ranasinghe, N., Pravar, J., Nguyen, T. N. T., Koh, R. K. C., Tolley, D., Karwita, S., Lin, L., Liangkun Y., Shamaiah K., Chow., E., Ching Chiuan, Y., Do, E.: Season Traveller: Multisensory Narration for Enhancing the Virtual Reality Experience: In: CHI '18: Proceedings of the 2018 CHI Conference on Human Factors in Computing Systems, pp. 1-13. ACM DL (2018).

8. VAQSO VR Device, https://vaqso.com/, last accessed 2017/01/16.

9. Multisensory VR mask Feelreal, https://feelreal.com/, last accessed 1999/02/15.

10. Papagiannis, H.: Augmented reality. Everything you wanted to know about future technology. Moscow: Eksmo (2019).

11. The Night Cafe - An Immersive VR Tribute to Vincent van Gogh, https://www.borrowedlightvr.com/the-night-cafe/, last accessed 2015/06/26. 